\title{
A model of adding two edges in levels of a complete binary tree
}

\author{
Kiyoshi Sawada ${ }^{1, *}$ \\ ${ }^{1}$ Department of Economic Information, University of Marketing and Distribution Sciences, Kobe, Japan
}

\begin{abstract}
This study proposes a model of adding two edges between nodes of the same level of a complete binary tree. Firstly we add one edge between the optimal two nodes with the optimal depth $\mathrm{N}^{*}$ maximizing a total shortening distance. Secondly we add another edge between nodes with the same depth $\mathrm{M}(\mathrm{M}=1,2, \ldots, \mathrm{H})$. The total shortening distance to obtain the optimal two nodes with the optimal depth $\mathrm{M}^{*}$ maximizing a total shortening distance is formulated.
\end{abstract}

\section{Introduction}

This paper proposes a new model of adding relations in an organization structure $[1,2]$. Relations between members in an organization are added to become effective in communication of information.

This study considers a pyramid organization structure which is a complete binary tree of height $H(H=2,3$, ...). A complete binary tree is a rooted tree in which all leaves have the same depth and all internal nodes have two children [3]. Nodes and edges in the complete binary tree correspond to units and relations between units in the organization. Moreover the path between a pair of nodes in the complete binary tree is equivalent to the route of communication of information between a pair of units in the organization, and adding edges is equivalent to forming additional relations other than that between each superior and his direct subordinates [4-7].

We have proposed a model [4-7] of adding one edge between two nodes with the same depth $N(N=1,2, \ldots$, $H$ ) of a complete binary tree. In this model we have obtained the optimal two nodes with the optimal depth $N^{*}$ maximizing a total shortening distance. The total shortening distance is the sum of shortened lengths of shortest paths between every pair of all nodes by adding edge between two nodes [4-7]. This means that the communication of information between every member in the organization becomes the most efficient.

This paper proposes a model of adding two edges between nodes of the same level of a complete binary tree. In this model firstly we add one edge between the optimal two nodes with the optimal depth $N^{*}$ maximizing a total shortening distance [4-7]. Secondly we add another edge between nodes with the same depth $M(M=$ $1,2, \ldots, H)$. We formulate the total shortening distance to

\footnotetext{
*Corresponding author: Kiyoshi_Sawada@red.umds.ac.jp
}

obtain the optimal two nodes with the optimal depth $M^{*}$ maximizing a total shortening distance.

\section{A model of adding one edge [4-7]}

This section formulates the total shortening distance when an edge between nodes with the same depth $N(N=$ $1,2, \ldots, H)$ in a complete binary tree of height $H(H=2$, $3, \ldots$ ) and obtains the optimal two nodes with the optimal depth $N^{*}$ maximizing a total shortening distance [4-7].

A new edge can be added between two nodes with the same depth $N$ in a complete binary tree in $N$ ways that lead to non-isomorphic graphs. Let $R_{H}(N, D)$ denote the total shortening distance by adding the new edge, where $D(D=0,1,2, \ldots, N-1)$ is the depth of the deepest common ancestor of the two nodes on which the new edge is incident. When the total shortening distance for the case of $D=0$ is denoted by $S_{H}(N)$, we obtain

$$
R_{H}(N, D)=S_{H-D}(N-D) .
$$

We formulate $S_{H}(N)$ in the following.

Let $v_{0}{ }^{X}$ and $v_{0}{ }^{Y}$ denote the two nodes on which the adding edge is incident and assume that $D=0$. Let $v_{k}^{X}$ and $v_{k}^{Y}$ denote ancestors of $v_{0}{ }^{X}$ and $v_{0}^{Y}$, respectively, with depth $N-k$ for $k=1,2, \ldots, N-1$. The sets of descendants of $v_{0}{ }^{X}$ and $v_{0}{ }^{Y}$ are denoted by $V_{0}{ }^{X}$ and $V_{0}{ }^{Y}$ respectively. Let $V_{k}^{X}$ denote the set obtained by removing the descendants of $v_{k-1}^{X}$ from the set of descendants of $v_{k}^{X}$ and let $V_{k}^{Y}$ denote the set obtained by removing the descendants of $v_{k-1}^{Y}$ from the set of descendants of $v_{k}^{Y}$, where $k=1,2, \ldots, N-1$.

The total shortening distance can be formulated by adding up the following three sums of shortening distances: (i) the sum of shortening distances between every pair of nodes in $V_{0}^{X}$ and nodes in $V_{0}^{Y}$, (ii) the sum 
of shortening distances between every pair of nodes in $V_{0}^{X}$ and nodes in $V_{k}^{Y}(k=1,2, \ldots, N-1)$ and between every pair of nodes in $V_{0}^{Y}$ and nodes in $V_{k}^{X}(k=1,2, \ldots$, $N-1$ ) and (iii) the sum of shortening distances between every pair of nodes in $V_{k}^{X}(k=1,2, \ldots, N-1)$ and nodes in $V_{k}^{Y}(k=1,2, \ldots, N-1)$.

The sum of shortening distances between every pair of nodes in $V_{0}{ }^{X}$ and nodes in $V_{0}{ }^{Y}$ is given by

$$
A_{H}(N)=[W(H-N)]^{2}(2 N-1),
$$

where $W(h)$ denotes the number of nodes of a complete binary tree of height $h(h=0,1,2, \ldots)$. The sum of shortening distances between every pair of nodes in $V_{0}{ }^{X}$ and nodes in $V_{k}^{Y}(k=1,2, \ldots, N-1)$ and between every pair of nodes in $V_{0}{ }^{Y}$ and nodes in $V_{k}^{X}(k=1,2, \ldots, N-1)$ is given by

$$
B_{H}(N)=2 W(H-N) \sum_{i=1}^{N-1}[W(H-i-1)+1](2 i-1),
$$

and the sum of shortening distances between every pair of nodes in $V_{k}^{X}(k=1,2, \ldots, N-1)$ and nodes in $V_{k}^{Y}(k=$ $1,2, \ldots, N-1)$ is given by

$$
\begin{aligned}
C_{H}(N) & =\sum_{i=1}^{N-2}[W(H-i-2)+1] \\
& \times \sum_{j=1}^{i}[W(H-N+j-1)+1](2 i-2 j+1),
\end{aligned}
$$

where we define $\sum_{i=1}^{0} \bullet=0$ and $\sum_{i=1}^{-1} \bullet=0$.

From Equations (2), (3) and (4), the total shortening distance $S_{H}(N)$ for the case of $D=0$ is formulated by

$$
S_{H}(N)=A_{H}(N)+B_{H}(N)+C_{H}(N) .
$$

From Equations (1) and (5) we have $R_{H}(N, D)$. We have Theorem 1 since

$$
R_{H}(N, D+1)-R_{H}(N, D)<0 .
$$

Theorem 1. $D^{*}=0$ maximizes $R_{H}(N, D)$ for each $N$ [47].

Theorem 1 shows that the most efficient way of forming relations to two members in each level is that to two members which doesn't have common superiors except the top.

We have Theorem 2 since

(i) When $H=2, S_{H}(2)-S_{H}(1)<0$, and

(ii) When $H=3,4, \ldots, S_{H}(2)-S_{H}(1)>0$ and $S_{H}(N+1)$ $-S_{H}(N)<0$ for $N=2,3, \ldots, H-1$.

Theorem 2. [4-7]

(i) If $H=2$, then $N^{*}=1$.

(ii) If $H=3,4, \ldots$, then $N^{*}=2$.

\section{A model of adding two edges}

This section proposes a new model of adding two edges between nodes of the same level of a complete binary tree. Firstly we add one edge between the optimal two nodes with the optimal depth $N^{*}$ in Section 2 of this paper. Secondly we add another edge between nodes with the same depth $M(M=1,2, \ldots, H)$. The total shortening distance to obtain the optimal two nodes with the optimal depth $M^{*}$ maximizing a total shortening distance is formulated in this section.

Let $n^{X}$ and $n^{Y}$ denote the two nodes on which the optimal first adding edge with the optimal depth $N^{*}$ is incident. Let $m^{X}$ and $m^{Y}$ denote the two nodes on which the second adding edge with the depth $M$ is incident. Let $\operatorname{DDCA}(u, v)$ denote the depth of the deepest common ancestor of node $u$ and node $v$.

The total shortening distance is formulated in the following cases.

(I) When $H=2, N^{*}=1$ and $M^{*}=2$ trivially.

(a) When $\operatorname{DDCA}\left(m^{X}, m^{Y}\right)=0$, the total shortening distance is 2 .

(b) When $\operatorname{DDCA}\left(m^{X}, m^{Y}\right)=1$, the total shortening distance is 1 .

(II) When $H=3,4, \ldots, N^{*}=2$.

(a) When $\operatorname{DDCA}\left(m^{X}, m^{Y}\right)=0$ and

(i) When $\operatorname{DDCA}\left(n^{X}, m^{X}\right)=1$ and $\operatorname{DDCA}\left(n^{Y}, m^{Y}\right)=$ 1, let $T_{1, H}(M)$ denote the total shortening distance for $M$ $=1,2, \ldots, H$.

(ii) When $\operatorname{DDCA}\left(n^{X}, m^{X}\right)=1$ and $\operatorname{DDCA}\left(n^{Y}, m^{Y}\right)=$ 2 or $\operatorname{DDCA}\left(n^{X}, m^{X}\right)=2$ and $\operatorname{DDCA}\left(n^{Y}, m^{Y}\right)=1$, let $T_{2, H}$ ( $M$ ) denote the total shortening distance for $M=2,3, \ldots$, $H$.

(iii) When $\operatorname{DDCA}\left(n^{X}, m^{X}\right)=2$ and $\operatorname{DDCA}\left(n^{Y}, m^{Y}\right)$ $=2$, let $T_{3, H}(M)$ denote the total shortening distance for $M=3,4, \ldots, H$.

(b) When $\operatorname{DDCA}\left(m^{X}, m^{Y}\right)>0, \operatorname{DDCA}\left(m^{X}, m^{Y}\right)=1$ maximizes the total shortening distance for each $M$ from Theorem 1 of Section 2. When $\operatorname{DDCA}\left(m^{X}, m^{Y}\right)=1$, let $T_{4, H}(M)$ denote the total shortening distance for $M=2$, $3, \ldots, H$.

$T_{1, H}(M)$ is given by

$$
T_{1, H}(M)=[W(H-2)+1]^{2},
$$

for $M=1$, and

$$
\begin{aligned}
T_{1, H}(M) & =[W(H-M)]^{2}(2 M-1) \\
& +2 W(H-M) \sum_{i=1}^{M-2}[W(H-i-2)+1](2 i+1) \\
& +2 W(H-M)+\sum_{i=1}^{M-2}[W(H-i-2)+1] \\
& \times \sum_{j=1}^{i}[W(H-M+j-1)+1](2 i-2 j+1),
\end{aligned}
$$

for $M=2,3, \ldots, H$.

$T_{2, H}(M)$ is given by 


$$
\begin{aligned}
T_{2, H}(M) & =[W(H-M)]^{2}(2 M-2) \\
& +2 W(H-M) \sum_{i=1}^{M-2}[W(H-i-2)+1] 2 i \\
& +W(H-M)[W(H-2)+1] \\
& +\sum_{i=1}^{M-3}[W(H-i-3)+1] \\
& \times \sum_{j=1}^{i}[W(H-M+j-1)+1](2 i-2 j+2),
\end{aligned}
$$

for $M=2,3, \ldots, H$.

$T_{3, H}(M)$ is given by

$$
\begin{aligned}
T_{3, H}(M)= & {[W(H-M)]^{2}(2 M-4) } \\
& +2 W(H-M) \sum_{i=1}^{M-3}[W(H-i-3)+1] 2 i \\
& +\sum_{i=1}^{M-4}[W(H-i-4)+1] \\
& \times \sum_{j=1}^{i}[W(H-M+j-1)+1](2 i-2 j+2),
\end{aligned}
$$

for $M=3,4, \ldots, H$.

$T_{4, H}(M)$ is given by

$$
\begin{aligned}
T_{4, H}(M)= & {[W(H-M)]^{2}(2 M-3) } \\
& +2 W(H-M) \sum_{i=1}^{M-2}[W(H-i-2)+1](2 i-1) \\
& +\sum_{i=1}^{M-3}[W(H-i-3)+1] \\
& \times \sum_{j=1}^{i}[W(H-M+j-1)+1](2 i-2 j+1),
\end{aligned}
$$

for $M=2,3, \ldots, H$.

\section{References}

1. S.P. Robbins, Essentials of Organizational Behavior, 7th Edition, Prentice Hall, Upper Saddle River, N.J. (2003)

2. Y. Takahara, M. Mesarovic, Organization Structure: Cybernetic Systems Foundation, Kluwer Academic / Plenum Publishers, New York (2003)

3. T.H. Cormen, C.E. Leiserson, R.L. Rivest, C. Stein, Introduction to Algorithms, 2nd Edition, MIT Press, Cambridge, Mass. (2001)

4. K. Sawada, H. Uno, Transactions of the Japan Society for Industrial and Applied Mathematics, 10, 335-346 (2000)

5. K. Sawada, Proceedings of 18th International Conference on Production Research (ICPR), Salerno, Italy (2005)

6. K. Sawada, R. Wilson, European Journal of Operational Research, 174, 1491-1500 (2006)

7. K. Sawada, Proceedings of the 8th WSEAS International Conference on System Science and Simulation in Engineering, Genova, Italy, 215-218 (2009) 